\title{
Artigo/Article
}

\section{Tuberculose em indígenas menores de 15 anos, no Estado de Mato Grosso do Sul}

\author{
Tuberculosis among Brazilian indigenous individuals aged less than 15 years-old in State of \\ Mato Grosso do Sul, Brazil
}

\section{Ana Maria Campos Marques ${ }^{1}$, Maurício Antonio Pompilio² ${ }^{2}$, Sandra Christo dos Santos ${ }^{1}$, Silvio Jacks dos Anjos Garnês $^{3}$ e Rivaldo Venâncio da Cunha ${ }^{2}$}

\section{RESUMO}

Introdução $\mathrm{O}$ objetivo do estudo foi descrever os aspectos epidemiológicos da tuberculose na população indígena com idade inferior a quinze anos, de Mato Grosso do Sul, Brasil, no período de 2000 a 2006, após a implantação do Subsistema de Saúde Indígena. Métodos: Estudo descritivo, retrospectivo, de base de dados secundários, utilizando-se o Banco de dados do Distrito Sanitário Especial Indígena de Mato Grosso do Sul e do Sistema de Informações de Agravos de Notificação. Variáveis analisadas: frequência por grupo etário, distribuição por sexo, forma clínica e desfecho dos casos. Teste de Fischer e curva de tendência para incidência, $\mathrm{p}<0,01$. Resultados: A proporção de casos de tuberculose em indígenas com idade inferior a 15 anos foi de 20,4\% (224/1.096). Verificou-se elevados coeficientes de incidência de tuberculose em indígenas com menos de 15 anos de idade, porém com curva descendente e uma queda anual em torno de $14 \%$. Houve predomínio em indivíduos com idade inferior a 5 anos e elevado número de casos com idade inferior a 1 ano. Mais da metade dos casos era do sexo masculino e a forma clínica pulmonar ocorreu em 92,9\%. A maioria (91,1\%) dos casos evoluiu para cura, $3,6 \%$ abandonaram o tratamento e 2,2\% evoluíram para óbito. Conclusões: A elevada taxa de cura, a reduzida mortalidade e a progressiva queda de incidência da doença no grupo etário inferior a 15 anos apontam para a efetividade da estratégia do tratamento supervisionado da tuberculose, no contexto do novo modelo de assistência à saúde indígena implantado no ano de 2000.

Palavras-chaves: Tuberculose. Crianças. Índios sul-Americanos.

\begin{abstract}
Introduction: This study describes the epidemiological aspects of TB among Brazilian Native Indians aged less than 15 years-old in Mato Grosso do Sul, Brazil, between 2000 and 2006. Methods: A retrospective observational study based on secondary data collected from the health system of Brazilian indigenous peoples, Special Indigenous Sanitary District (DSEI), and National System of Notifiable Diseases (SINAN) databases. The variables analyzed were: frequency of TB according to sex, age, clinical presentation and outcome. The Fisher test and the incidence tendency curve were calculated $(\mathrm{p}<0.01)$. Results: TB prevalence was $20.4 \%$ $(224 / 1,096)$. The incidence rate decreased $14 \%$ per year during the study period. TB was more prevalent among Brazilian indigenous individuals aged less than 5 years-old and among those aged less than one year-old. More than half of TB cases were male and the most common clinical presentation was pulmonary TB (92.9\%). Cure was the most common outcome (91.1\%), followed by abandoned treatment (3.6\%) and death (2.2\%). Conclusions: The high cure rate, reduced mortality and the progressive decrease in TB incidence rate during the study period indicate the effectiveness of supervised treatment of the new TB control model implemented among Brazilian indigenous peoples on 2000.
\end{abstract}

Key-words: Tuberculosis. Children. South American indigenous peoples.

1. Curso de Medicina, Universidade Anhanguera Uniderp, Campo Grande, MS. 2. Departamento de Clínica Médica, Faculdade de Medicina, Universidade Federal de Mato Grosso do Sul, Campo Grande, MS. 3. Departamento de Engenharia Cartográfica, Setor de Tecnologia e Geociências, Universidade Federal de Pernambuco, Recife, PE.

Endereço para correspondência: Dra. Ana Maria Campos Marques. Rua Treze de Junho 1651/1501, 79002-430 Campo Grande, MS.

Tel: 5567 3026-8044

e-mail: anamaria@ronimarques.med.br

Recebido para publicação em 30/04/2010

Aceito em 13/07/2010

\section{INTRODUÇÃO}

O Brasil está entre os vinte e dois países que detêm $90 \%$ dos casos de tuberculose (TB) no mundo $^{1}$ e, anualmente, notifica cerca de 72.000 casos novos (incidência), com uma média de 38,2 casos por 100 mil habitantes ${ }^{2}$. Os dados nacionais são insuficientes para a compreensão da situação epidemiológica da TB entre os povos indígenas. A incidência da tuberculose nessas populações é elevada e, algumas vezes, os percentuais são dez vezes superiores aos encontrados na população brasileira em geral ${ }^{3}$. A população indígena no Brasil está mais concentrada nos Estados do Amazonas, Mato Grosso do Sul, Mato Grosso e Roraima ${ }^{4}$. $\mathrm{O}$ alto percentual de tuberculose em menores de 15 anos é de grande importância epidemiológica e representa um evento sentinela em Saúde Coletiva, visto que se refere à infecção recente, promovida por contato com bacilífero. Esse fato foi destacado por Marques e Cunha ${ }^{5}$, no estudo em que constataram que esse grupo etário representava $40 \%$ dos doentes por tuberculose, em relação aos pacientes com mais de 15 anos de idade 5 .

Deve-se esclarecer que, no Brasil, utilizam-se critérios epidemiológicos, clínicos e laboratoriais para diagnóstico da TB em menores de 15 anos, conforme normas do Programa Nacional de controle da Tuberculose (PNCT $)^{6}$. Com o avanço da implantação de um subsistema de atenção diferenciado, para a saúde indígena (Lei no 9836/1999), o Distrito Sanitário Especial Indígena (DSEI) emergiu como um modelo de organização de serviço destinado a esta população específica ${ }^{7}$. Historicamente, desde a década de 60, o Hospital e Maternidade Indígena Porta da Esperança (HPE), da Missão Evangélica Caiuá, uma entidade filantrópica no Município de Dourados, MS, era centro de assistência à saúde indígena e centro de referência para o diagnóstico e tratamento da TB, na população indígena do estado. Esse modelo se manteve até o ano de 2000 quando, com a implantação do subsistema em MS, a atenção à saúde indígena foi descentralizada e passou a ser coordenada por 13 pólos-base, que compõem o 
DSEI-MS, sob a responsabilidade das equipes de saúde indígena atuantes nas aldeias. Essas equipes, quando assumiram as ações de controle da TB, priorizaram o tratamento diretamente observado por agentes de saúde indígenas. Por conseguinte, são fatores que justificam este estudo as peculiaridades demográficas da população indígena do estado, as mudanças no modelo de assistência à saúde dessa população a partir de 2000, a necessidade de avaliação regional que subsidie o planejamento das ações de saúde, e a importância da tuberculose em menores de 15 anos como indicador de controle da doença.

\section{MÉTODOS}

Trata-se de um estudo descritivo, retrospectivo, de levantamento de base de dados secundários. A população do estudo é indígena, menores de 15 anos residentes nas aldeias indígenas do Estado de Mato Grosso do Sul, Brasil, casos novos de tuberculose diagnosticados e registrados pelo sistema de saúde indígena no período de 2000 a 2006. Segundo dados da Fundação Nacional da Saúde/MS (FUNASA/MS), a população indígena do Mato Grosso do Sul era de 51.428 indivíduos em 2000 e de 58.502 em 2006, com, respectivamente, $52,2 \%$ e $49 \%$ menores de 15 anos de idade ${ }^{8}$. Os casos de tuberculose foram retirados do livro Registro e Controle do Tratamento dos Casos de Tuberculose, conforme as normas do PNCT/ MS, do Banco de dados do DSEI-MS. Houve concordância entre este banco de dados e o SINAN/SES-MS. Os dados referentes ao encerramento do caso que estivessem incompletos foram atualizados pelo Banco de dados do SINAN/SES-MS. Foram analisadas: a frequência por grupo etário, a distribuição por sexo, à forma clínica e o desfecho dos casos. Para a determinação dos coeficientes de incidência, foram utilizados os dados demográficos (população estimada por ano e baseada no censo 2000) fornecidos pelo DSEIMS, disponíveis no Sistema de Informações da FUNASA-MS. O Banco de dados foi tratado no programa Excel (Microsoft Office 2003).

\section{Ética}

Este estudo foi aprovado pelo Comitê de Ética em Pesquisa para Seres Humanos, da Universidade Anhanguera-UNIDERP, e pela Comissão Nacional de Ética em Pesquisa (CONEP).

\section{RESULTADOS}

Como mostrado na Figura 1, a proporção de casos de tuberculose em indígenas menores de 15 anos em Mato Grosso do Sul, no período do estudo, foi de 224, em um total de 1.096 (20,2\%) casos. Observase que a proporção de casos no ano 2000 aproximava-se de $40 \%$ nesse grupo etário, com progressiva redução até 2006 (10,9\%). Assim, os elevados coeficientes de incidência da doença em indígenas menores de 15 anos no período estudado apresentam uma curva descendente, com queda anual em torno de 14\% (Figura 2). A frequência por grupo etário revela predomínio em indivíduos com menos de 5 anos de idade ( $83 \%$ em 2002 e $70 \%$ em 2003 e 2004), bem como, no mesmo período, elevado número de casos em menores de 1 ano de idade (Tabela 1). O sexo masculino predominou com mais de 50\% dos casos, exceto em 2002 e 2006. A forma pulmonar (92,9\%) foi a predominante, enquanto a distribuição das formas extrapulmonares foi de $7,1 \%$, a ganglionar periférica (Gp) foi de $31,2 \%(5 / 16)$, a pleural (Pl) 25\% (4/16), a menigoencefalite (Me) 18,8\% (3/16), a miliar (Mi) $12,5 \%(2 / 16)$ e a osteoarticular (Oa) $12,5 \%(2 / 16)$ (Figura 3). Quanto ao desfecho, 91,1\% dos casos evoluíram para cura, 2,2\% para óbito e 3,6\% abandonaram o tratamento (Tabela 2).

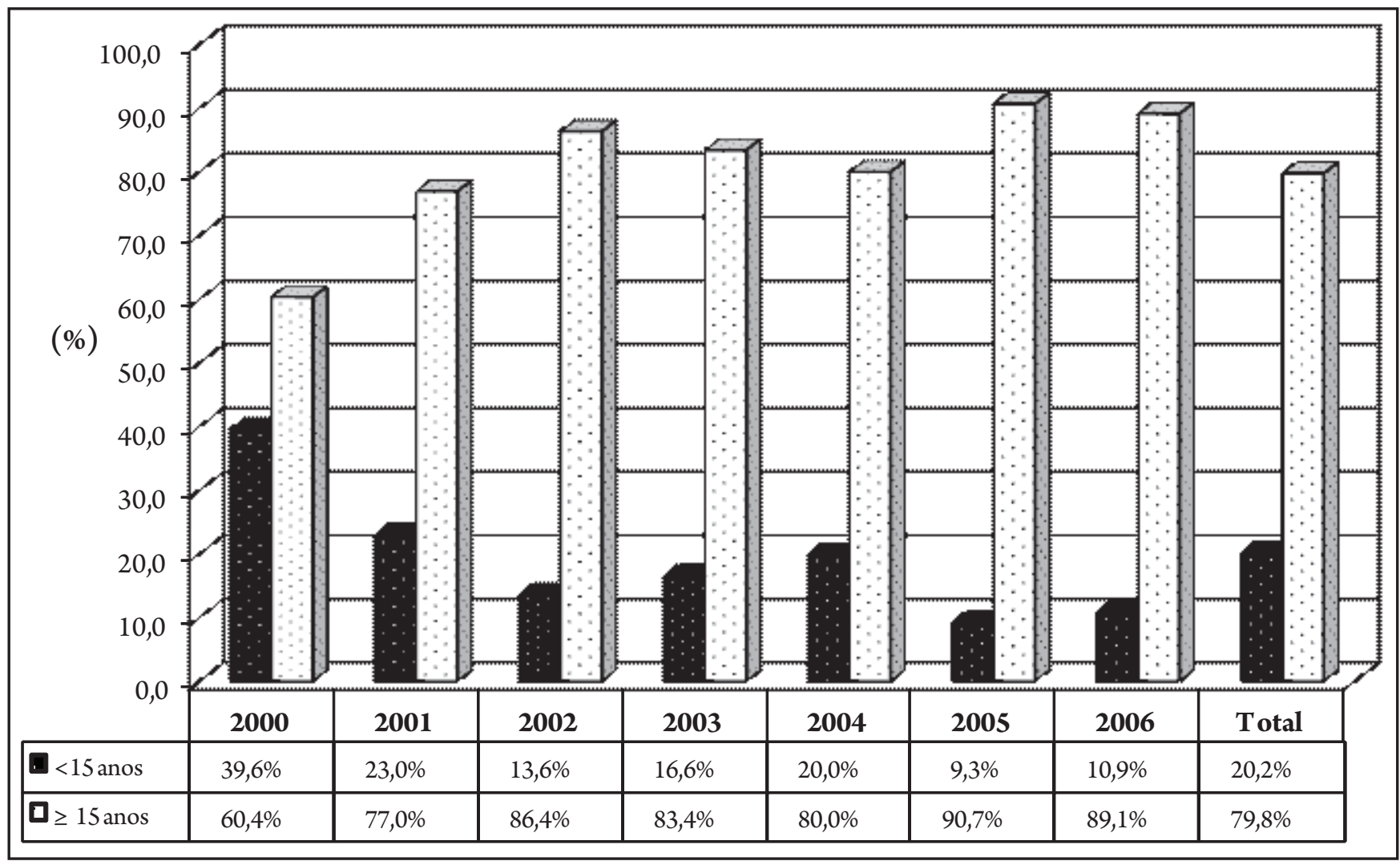

FIGURA 1 - Distribuição da proporção de casos de tuberculose em menores de 15 anos em relação aos maiores ou iguais a 15 anos, na população indígena de Mato Grosso do Sul, 2000 a 2006. 


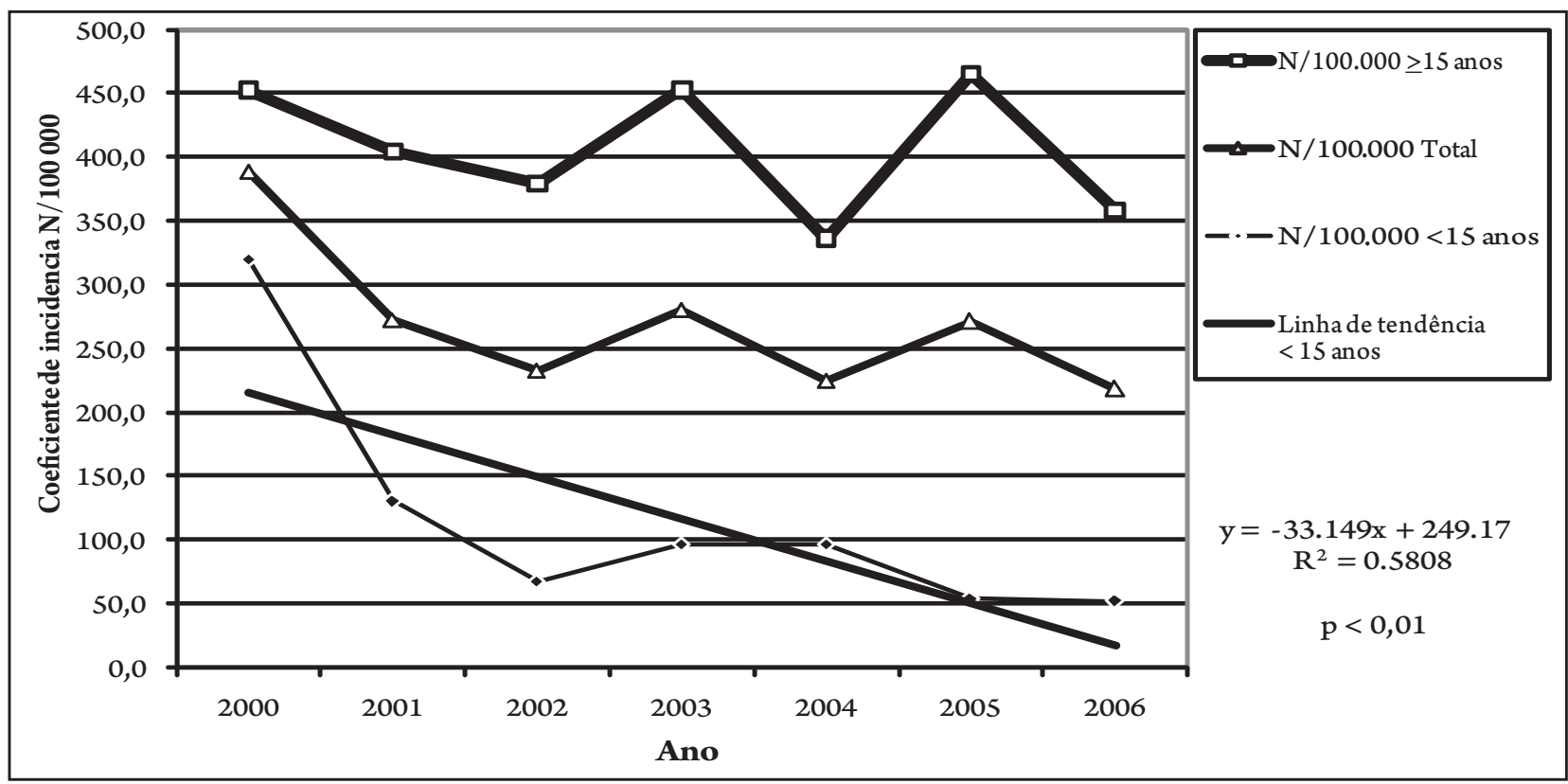

FIGURA 2 - Coeficiente de incidência de tuberculose por 100.000 habitantes em indígenas menores de 15 anos, maiores ou iguais a 15 anos, na população indígena geral de Mato Grosso do Sul, 2000 a 2006.

TABELA 1 - Distribuição do número e frequência de casos de tuberculose por grupo etário e sexo em menores de 15 anos na população indígena de Mato Grosso do Sul, 2000 a 2006.

\begin{tabular}{|c|c|c|c|c|c|c|c|c|c|c|c|c|c|}
\hline \multirow[b]{3}{*}{ Ano } & \multicolumn{9}{|c|}{ Grupo etário (anos) } & \multicolumn{4}{|c|}{ Sexo } \\
\hline & \multicolumn{2}{|c|}{$<1$} & \multicolumn{2}{|c|}{1 a 4} & \multicolumn{2}{|c|}{$5 \times 9$} & \multicolumn{2}{|c|}{10 a 14} & \multirow{2}{*}{$\frac{\text { total }<15}{\mathrm{n}^{\mathrm{o}}}$} & \multicolumn{2}{|c|}{ feminino } & \multicolumn{2}{|c|}{ masculino } \\
\hline & $\mathrm{n}^{\mathrm{o}}$ & $\%$ & $\mathrm{n}^{\mathrm{o}}$ & $\%$ & $\mathrm{n}^{\mathrm{o}}$ & $\%$ & $\mathrm{n}^{\mathrm{o}}$ & $\%$ & & $\mathrm{n}^{\mathrm{o}}$ & $\%$ & $\mathrm{n}^{\mathrm{o}}$ & $\%$ \\
\hline 2000 & 6 & 7,0 & 37 & 43,0 & 29 & 33,7 & 14 & 16,3 & 86 & 42 & 48,8 & 44 & 51,2 \\
\hline 2001 & 5 & 14,3 & 13 & 37,1 & 8 & 22,9 & 9 & 25,7 & 35 & 16 & 45,7 & 19 & 54,3 \\
\hline 2002 & 4 & 22,2 & 11 & 61,1 & 1 & 5,6 & 2 & 11,1 & 18 & 13 & 72,2 & 5 & 27,8 \\
\hline 2003 & 4 & 14,8 & 16 & 59,3 & 6 & 22,2 & 1 & 3,7 & 27 & 11 & 40,7 & 16 & 59,3 \\
\hline 2004 & 5 & 18,5 & 14 & 51,9 & 3 & 11,1 & 5 & 18,5 & 27 & 11 & 40,7 & 16 & 59,3 \\
\hline 2005 & 1 & 6,3 & 6 & 37,5 & 3 & 18,8 & 6 & 37,5 & 16 & 7 & 43,8 & 9 & 56,3 \\
\hline 2006 & 1 & 6,7 & 7 & 46,7 & 4 & 26,7 & 3 & 20,0 & 15 & 10 & 66,7 & 5 & 33,3 \\
\hline Total & 26 & 11,6 & 104 & 46,4 & 54 & 24,1 & 40 & 17,9 & 224 & 110 & 49,1 & 114 & 50,9 \\
\hline
\end{tabular}

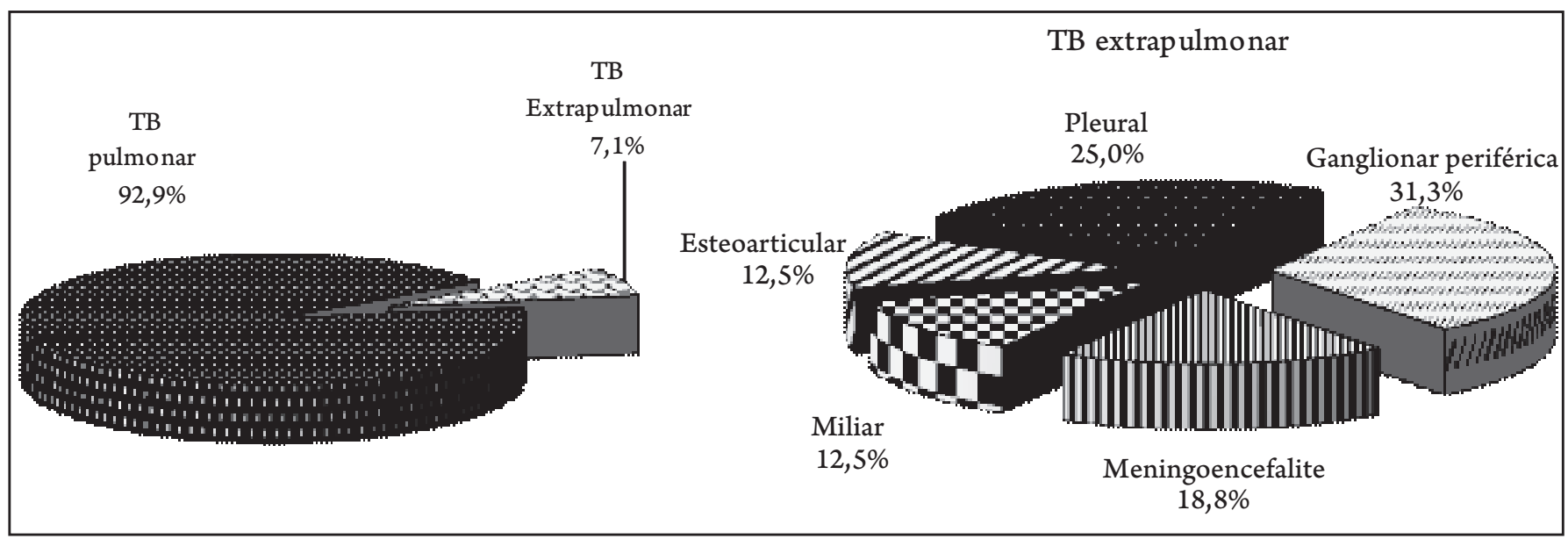

FIGURA 3 - Distribuição dos percentuais dos casos de tuberculose, segundo a forma clínica em menores de 15 anos na população indígena de Mato Grosso do Sul, 2000 a 2006. 
TABELA 2 - Distribuição dos casos de tuberculose segundo o desfecho em menores de 15 anos na população indígena de Mato Grosso do Sul, 2000 a 2006.

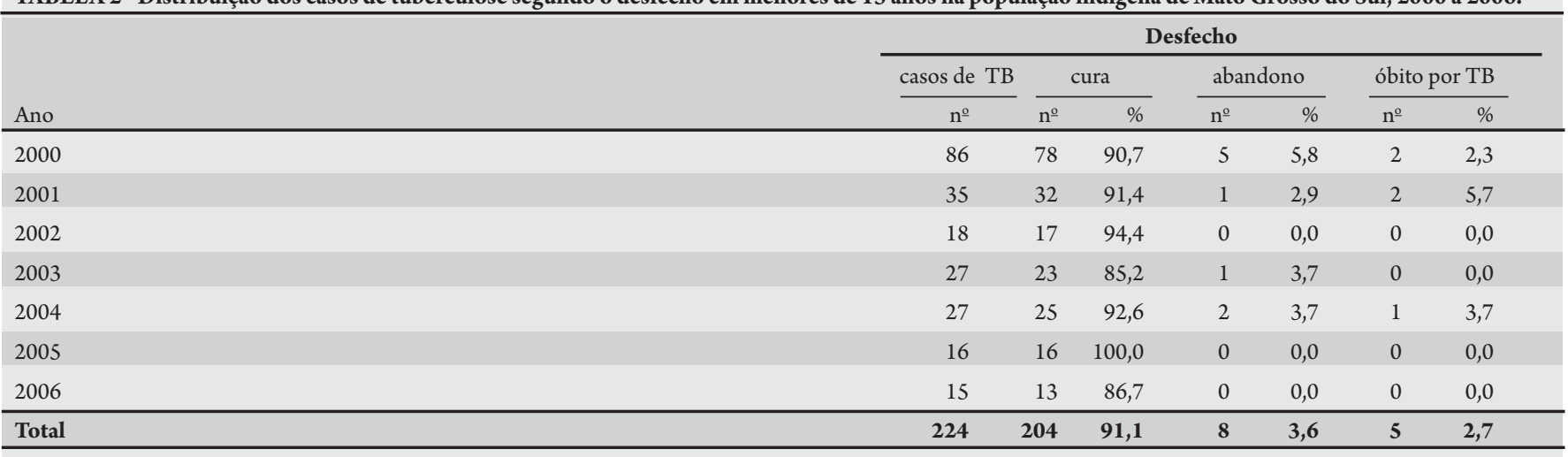

TB: tuberculose.

\section{DISCUSSÃO}

Embora apresente as limitações próprias dos levantamentos de dados secundários, esta pesquisa constitui importante ferramenta de conhecimento capaz de problematizar e subsidiar a gestão de serviços de saúde. A descentralização dos sistemas de informação em saúde e do gerenciamento do SINAN TB é extremamente importante, porém se observa situações de registro incompleto de dados bem como subnotificação ${ }^{9-11}$. Entre as variáveis pesquisadas no período de 2000 a 2006 houve alto grau de completude. Os resultados deste estudo foram obtidos após a implantação do subsistema de atenção à saúde indígena e o estabelecimento do DSEI-MS e, portanto, após a mudança de estratégia no controle da doença nesta população vulnerável. A proporção de casos em indivíduos menores de 15 anos, que no ano 2000 aproximava-se de 40\%, apresentou progressiva redução até 2006 $(10,9 \%)$. Essa evolução, bem como a proporção de $20,4 \%$ desse grupo etário, no total de casos, aponta para uma redução da incidência da doença nessa população no período estudado. Se considerarmos a taxa de $40 \%$ verificada no estudo de Marques e Cunha ${ }^{5}$, na mesma população, no período de 1996 a 19995, antes da implantação do subsistema de saúde indígena, pode-se evidenciar o impacto positivo do novo modelo de assistência. Ainda assim, tal resultado supera a proporção de $16 \%$ dos casos em menores de 15 anos na população indígena nacional, no período de 2003 a $2007^{12}$, e está acima dos $5 \%$ esperados para essa faixa etária, na população brasileira ${ }^{13}$. Contudo, esses dados são positivos, em relação aos $45 \%$, encontrado em indígenas Surui na Amazônia, de 1991 a $2002^{14}$, e aos 31,2\% verificados na população predominantemente indígena do Município de São Gabriel da Cachoeira, Estado do Amazonas, no período de 1997 a $2002^{15}$.

Aqueda do coeficiente de incidência em indígenas com menos de 15 anos de idade foi intensa, com um decréscimo anual em torno de $14 \%$, observando-se uma quebra dessa curva descendente em 2003 e 2004 . O coeficiente de incidência no ano 2000 era superior a 300 casos $/ 100.000$ e em dois anos caiu para uma taxa em torno de 70 casos/100.000 (2002), ascendendo de forma surpreendente para $100 \mathrm{casos} / 100.000$, em 2003 e 2004, e retomando a tendência de queda, alcançando 50 casos/100.000 em 2005 e 2006. Esse fato não foi verificado no coeficiente de incidência da população indígena total e nos indivíduos com mais de 15 anos de idade e, portanto, trata-se de um evento restrito aos menores de 15 anos. No mesmo período, também foi observado um elevado número de casos de tuberculose em menores de 1 ano.
Conforme o teste de Fisher, podemos concluir que existe uma relação entre a incidência de doença (TB) e a idade da população. $\mathrm{O}$ valor de $\mathrm{p}$ tem significância e há tendência decrescente desta incidência entre menores de 15 anos conforme demonstrada na curva (Figura 2).

Destaca-se que em 2003 e 2004, na região da Grande Dourados (Pólo-Base Dourados), houve elevada ocorrência de desnutrição em crianças indígenas, com elevado número de óbitos atribuídos a esta causa. É possível especular-se que tenha existido uma relação de causa e efeito na associação dos eventos desnutrição e aumento da taxa de TB em menores de 15 anos, com predomínio em menores de 1 ano. O coeficiente de incidência de 50 casos $/ 100.000$ verificado no final do período de estudo, ainda é superior ao encontrado na população geral do Brasil (38,2/100.000), na população do Estado do Mato Grosso do Sul $(36,33 / 100.0002007)^{2}$ e em não indígenas com menos de 15 anos de idade, encontradas no Rio de Janeiro, na década de $90(27 / 100.000)^{16}$. Houve predomínio em menores de 5 anos (70 a 80\%) com elevado número de casos em menores de 1 ano no período de 2002 a 2004. Não houve diferença significativa, entre sexos e a forma clínica predominante foi a pulmonar (92,9\%), com a ocorrência de apenas $7,1 \%$ de localização extrapulmonar. Esse resultado é inferior tanto ao estudo nacional de indígenas menores de 15 anos de São Gabriel da Cachoeira (85\% pulmonar e 15,7\% extrapulmonar $)^{15}$ como a outros relatos da literatura, que indicam que cerca de $20 \%$ dos casos de TB em crianças têm apresentação extrapulmonar ${ }^{17}$. No entanto, nas formas extrapulmonares observase um distribuição semelhante à da literatura ${ }^{16,17} \mathrm{com}$ maior frequência da forma $\mathrm{Gp}$ (31,3\%), e Pl (25\%), seguido de 18,8\% de $\mathrm{Me}, 12,5 \% \mathrm{Mi}$ e $12,5 \%$ osteoarticular (Oa). Quanto ao desfecho dos casos, os resultados foram favoráveis, com 91,1\% de cura, 3,6\% de abandono e 2,2\% de óbitos. Esses resultados superam os índices nacionais (cura 77\%, abandono 8\%) e regionais de Mato Grosso do Sul (cura 72,5\%, abandono 10\%, óbito 8\%) ${ }^{18,19}$. A taxa de cura superou a meta de $85 \%$ pactuada pelo PNCT com a OMS. Apesar da elevada incidência da TB na população estudada, os casos, uma vez diagnosticados, são assistidos pelas equipes de saúde indígenas, estratégia que aparentemente possibilitou o alto índice de cura.

Ainda que se considerem as limitações do presente estudo, que se fundamenta em dados secundários, associadas às peculiaridades culturais das informações demográficas indígenas, que resultam em menor precisão de registros, é possível inferir que a elevada 
taxa de cura e a reduzida mortalidade assinalam que a estratégia do tratamento supervisionado da TB é efetiva no contexto do novo modelo de assistência a saúde indígena implantado no ano de 2000. No entanto, a elevada incidência de tuberculose em menores de 15 anos, apesar de uma queda anual da ordem de 14\%, é um indicador indireto da transmissibilidade do bacilo nas aldeias, pois revela infecção recente por contato com bacilíferos. Isso sugere dificuldades no controle de contatos e pode-se afirmar que ainda é alto o risco de um indígena de Mato Grosso de Sul, com menos de 15 anos de idade adoecer por tuberculose. Os dados também sugerem que, para a correção de rumos e o efetivo controle da doença, é importante considerar a natureza multifatorial da tuberculose e seus determinantes sociais.

\section{AGRADECIMENTOS}

A todos os profissionais das equipes de saúde indígena pelo registro das informações e em especial a Enfermeira Rosilene Lopes de Oliveira Figueiredo, sempre solicita em nos fornecer os dados. Á enfermeira Marli Marques, Gerente Técnica do PCT-PCH/SES/MS por disponibilizar os dados do SINAM-TB de Mato Grosso do Sul.

\section{CONFLITO DE INTERESSE}

Os autores declaram não haver nenhum tipo de conflito de interesse no desenvolvimento do estudo.

\section{SUPORTE FINANCEIRO}

Este estudo recebeu apoio financeiro do Conselho Nacional de Pesquisas (CNPq), processo 401.234/2005-3.

\section{REFERÊNCIAS}

1. Global Tuberculosis Control: a short update to the 2009 report.[Internet]. World Health Organization. 2009 - [acessado em 23 de março de 2010]. Disponível em: <http://www.who.int/tb/publications/global_report/2009/pdf/full.pdf>.

2. Ministério da Saúde. Situação da Tuberculose no Brasil - Programa Nacional de Controle da Tuberculose. Secretaria de Vigilância em Saúde, Departamento de Vigilância Epidemiológica. 2009- [acessado em 23 de março de 2010]. Disponível em: $<$ http://portal.saude.gov.br/portal/arquivos/pdf/apresentacao_tb_2009.pdf>.

3. Ministério da Saúde. Programa Nacional de Controle da Tuberculose. Plano Estratégico para o Controle da Tuberculose, Brasil 2007-2015. Brasília: Secretaria de Vigilância em Saúde, Departamento de Vigilância Epidemiológica; 2006. p.13.

4. Ministério da Saúde. Política Nacional de Atenção à Saúde dos Povos Indígenas. Brasília; 2000.

5. Marques AMC, Cunha RV. A medicação assistida e os índices de cura de tuberculose e de abandono de tratamento na população indígena Guaraní-Kaiwá no Município de Dourados, Mato Grosso do Sul, Brasil. Cad Saude Publica 2003; 19 (supl 5):1405-1411.

6. Ministério da Saúde. Manual de normas para o controle da tuberculose. J Pneumol 1997; 23:281-293.

7. Mendes EV. Distrito Sanitário: O processo social de mudança das práticas sanitárias no Sistema Único de Saúde. $2^{\text {a }}$ edição. São Paulo: Hucitec/ABRASCO; 1993. p.159-186.

8. Ministério da Saúde. Saúde Indígena. Demografia dos Povos Indígenas. [Internet]. Fundação Nacional de Saúde. [Acessado em fevereiro de 2010]. Disponível em <http://www.funasa.gov.br/internet/desai/sistemaSiasiDemografia Indigena.asp $>$.
9. Nogueira JA, Sá LD, França UM, Almeida SA, Lima DS, Figueiredo TMRM, et al. O sistema de informação e o controle da tuberculose nos municípios prioritários da Paraíba - Brasil. Rev Esc Enferm USP 2009; 43:125-131.

10. Branco MAF. Sistemas de informação em saúde no nível local. Cad Saude Publica 1996; 12:267-270 1996

11. Coelho DMM, Viana RL, Madeira CA, Ferreira LOC, Campelo V. Perfil epidemiológico da tuberculose no Município de Teresina-PI, no período de 1999 a 2005. Epidemiol Serv Saúde 2010; 19:34-43.

12. Ministério da Saúde. Estratégia para o controle da Tuberculose em população indígena. Programa Nacional de Controle da Tuberculose. [Internet]. Secretaria de Vigilância em Saúde, Departamento de Vigilância Epidemiológica. 2009. [acessado em 23 de março de 2010]. Disponível em <http://portal.saude.gov. $\mathrm{br} /$ portal/arquivos/pdf/estrategia_controletb_2009.pdf $>$.

13. Ministério da Saúde. Guia de vigilância epidemiológica. $7^{\text {a }}$ ed. Brasília: Secretaria de vigilância em Saúde, Departamento de Vigilância Epidemiológica; 2009. p. 54.

14. Basta-Jr PC, Coimbra CEA, Escobar AL, Santos RV. Aspectos epidemiológicos da tuberculose na população indígena Suruí, Amazônia, Brasil. Rev Soc Bras Med Trop 2004; 37 (supl 4):338-342.

15. Levino A, Oliveira RM. Tuberculose na população indígena de São Gabriel da Cachoeira, Amazonas, Brasil. Cad Saude Publica 2007; 23 (supl 7): 1728-1732.

16. Alves R, Sant'anna CC, Cunha AJLA. Epidemiologia da tuberculose infantil na cidade do Rio de Janeiro, RJ. Rev Saude Publica 2000; 34 (supl 4):409-410.

17. Le Roux P, Quinque K, Bonnel AS, Le Luyer B. Extrapulmonary tuberculosis in childhood. Arch Pediatr 2005; 12 (supp 1):122-126.

18. Fundação Nacional de Saúde. Plano Nacional de Controle da Tuberculose Normas técnicas: estrutura e operacionalização. Brasília: Ministério da Saúde; 2000 .

19. Informe Epidemiológico do Programa de Eliminação da Hanseníase e Controle da Tuberculose. Situação Epidemiológica da Tuberculose no Mato Grosso do Sul, Campo Grande 2006; 1:3-4. 